\title{
Damage by Induced Electric Field in Beam-sensitive Materials
}

Nan Jiang ${ }^{1}$

${ }^{1 .}$ Department of Physics, Arizona State University, Tempe AZ.

Beam damage limits the experimental resolution of state-of-the-art (scanning) transmission electron microscopy ((S)TEM) [1]. Despite its importance, our understanding of damage mechanisms has not been as highly valued as it should be. Both knock-on damage and radiolytic process, the mostly referred mechanisms so far, produce atomic displacements randomly. Therefore, they cannot directly interpret some common damage phenomena observed in beam-sensitive materials, such as phase separation and phase transformation. In these types of damage, it appears that a type of swarm behavior causes the same species of atoms to move together coherently [2]. Here, I will review recently modified mechanism, damage by the induced electric field (DIEF), and apply this mechanism to interpret damage phenome in beam-sensitive materials.

In DIEF mechanism, the charging process in a beam-transparent specimen is initiated by the excitations of atomic electrons, and the density of these excitations increases with the increase of current density of electron beam. As long as holes created by the emissions of secondary and Auger electrons cannot be neutralized immediately, positive charges can be trapped in the illuminated region. The buildup of positive charges may suppress the further emissions. Therefore $\mathrm{dQ}_{\mathrm{E}}(\mathrm{t}) / \mathrm{dt}$ decreases with the increase of illumination time, assuming $\mathrm{Q}_{\mathrm{E}}(\mathrm{t})$ is emitted electrons at time $\mathrm{t}$. By contrast, if $\mathrm{Q}_{\mathrm{A}}(\mathrm{t})$ represents charges been attracted into the illuminated region by the buildup of positive charges, $\mathrm{dQ}_{\mathrm{A}}(\mathrm{t}) / \mathrm{dt}$ increases initially with the increase of illumination. At time $t_{0}, d_{\mathrm{E}}(\mathrm{t}) / \mathrm{dt}=\mathrm{dQ}_{\mathrm{A}}(\mathrm{t}) / \mathrm{dt}$. Therefore, $\mathrm{t}_{0}$ can be considered as the equilibrium time, at which the charge accumulation reaches the maximum. Accordingly, the electric field induced by these charges $\mathrm{E}(\mathrm{t})$ also increases with the increase of illumination until it reaches the maximum strength $E_{\max }$ at time $t_{0}$. It should be pointed out that $t_{0}$ should be dependent on both beam current density and the settling time $\tau$ of the charge produced by the excitation. The latter is proportional to the resistivity of the specimen.

To damage specimen, the field $\mathrm{E}$ must be strong enough to displace atoms. Therefore, the work done by the field on the displaced atom as it moves from one site to the nearest available site must be larger than its activation energy for migration $U_{\mathrm{a}}$. Therefore, there is threshold field strength, $\mathrm{E}^{\mathrm{Th}}=U_{a} / q d$, such that damage occurs only if the induced electric field is stronger than this threshold. Here $q$ is the net charge of displaced atom, which is the difference between the number of valence electrons of the neutral atom and the number of electrons it carries in a bond or bonds, and $d$ is the shortest distance that the atom can be displaced. The value $d$ is approximately the cation-cation or anion - anion distance, e.g. $<1 \mathrm{~nm}$.

The mechanism of DIEF is schematically described in Fig. 1. In this mechanism, there is an intrinsic threshold $\mathrm{E}^{\mathrm{Th}}$, below which DIEF does not kick in. Above the $\mathrm{E}^{\mathrm{Th}}$ line is the DIEF regime, where the DIEF mechanism takes over and overshadows the effects of other mechanisms. $\mathrm{E}^{\mathrm{Th}}$ is determined by the physical and chemical properties of the material. The induced electric field E increases with increasing irradiation time at an initial exposure, and reaches a maximum at time $t_{0}$, when the charge accumulation rate becomes zero, i.e. $\mathrm{dQ} / \mathrm{dt}=0$. Therefore, there is a beam current density $\mathrm{J}^{\mathrm{Th}}$ for the maximum $\mathrm{E}\left(\mathrm{t}_{0}\right)=\mathrm{E}^{\mathrm{Th}}$, as shown in Fig. 1. If $J_{0}<J^{T h}, \mathrm{E}\left(\mathrm{t}_{0}\right)<\mathrm{E}^{\mathrm{Th}}$, so that the electric field is not strong enough to cause atomic displacement directly. If $J_{0}>J^{T h}, \mathrm{E}\left(\mathrm{t}_{0}\right)>\mathrm{E}^{\mathrm{Th}}$ and massive atomic displacements could occur. Therefore, 
$J^{T h}$ is the threshold beam current density, which can be measured by experiment [2]. However, damage does not occur immediately even though the current density $J_{0}>J^{T h}$. The induced electric field gradually increases with increasing irradiation until $t_{A}$, at which $\mathrm{E}\left(\mathrm{t}_{\mathrm{A}}\right)=\mathrm{E}^{\mathrm{Th}}$. Therefore, this is another experimental threshold, which is measured by the exposure time $t_{A}\left(J_{0}\right)$ for a given current density $J_{0}\left(>J^{T h}\right)$. At a given $J_{0}$, DIEF will not occur unless the exposure time is longer than the threshold $t_{A}\left(J_{0}\right)$, i.e. $t \geq t_{A}\left(J_{0}\right)$. This threshold depends on the current density; the higher the current density, the shorter the threshold exposure time is.

In conclusion, DIEF is electron dose-rate dependent. There are two thresholds for DIEF: a dose-rate threshold and an irradiation time threshold. For a dose rate lower than the threshold, DIEF does not operate alone, and thus knock-on and radiolysis may dominate. For a dose rate higher than the threshold, DIEF may take over, but is dependent on irradiation time. If the irradiation time is shorter than the time threshold (incubation period), DIEF does not occur either. DIEF kicks in only when the irradiation time is longer than the threshold time.

\section{References:}

[1] N. Jiang, Report on Progress in Physics 79 (2016), p. 016501.

[2] N. Jiang, J. Phys D: Appl. Phys. 46 (2013), p. 305502.

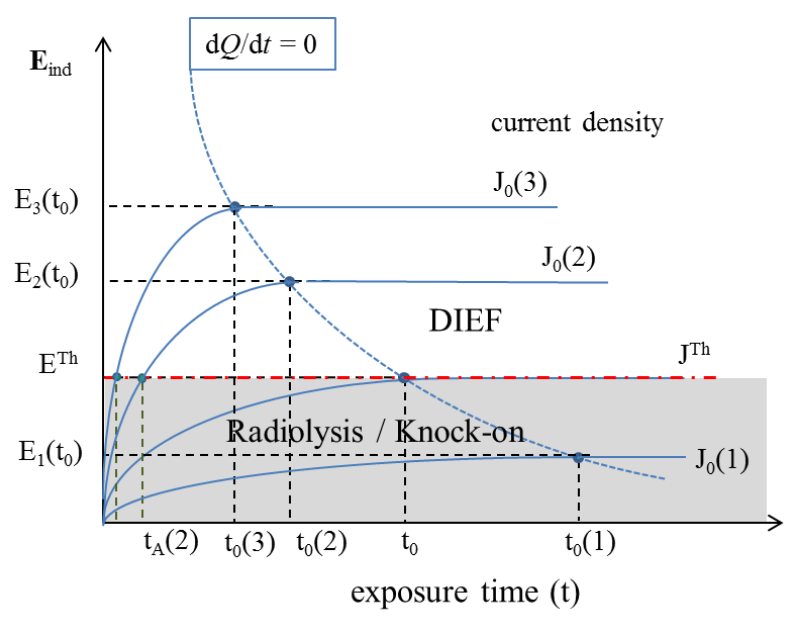

Figure 1. Schematic drawing of the DIEF model. DIEF only occurs if the induced electric field is stronger than the threshold. Below the threshold, it is radiolysis/knock-on regime. Beam current density $\mathrm{J}_{0}(3)>$ $\mathrm{J}_{0}(2)>\mathrm{J}^{\mathrm{Th}}>\mathrm{J}_{0}(1)$, in which $\mathrm{J}^{\mathrm{Th}}$ is the threshold beam current density. The intersection of each $\mathrm{J}_{0}(\mathrm{i})$ curve with $E^{\text {Th }}$ gives the threshold of exposure time for this current density $J_{0}(i)$. 